\title{
ORIGINAL
}

\section{SALUD ORAL DE LOS ESCOLARES DE CEUTA. INFLUENCIAS DE LA EDAD, EL GÉNERO, LA ETNIA Y EL NIVEL SOCIOECONÓMICO}

Víctor Miguel Nieto García (1), María Adoración Nieto García (2), Juan Ramón Lacalle Remigio (2) y Laila Abdel-Kader Martín (2)

(1) Instituto Nacional de la Salud, Ceuta

(2) Facultad de Medicina, Universidad de Sevilla

\section{RESUMEN}

Fundamento: La caries dental tiene una etiología multifactorial en la que intervienen características del huésped (saliva y esmalte dental), de la flora bucal (placa bacteriana) y del substrato sobre el que ésta se desarrolla (higiene oral y dieta). El objetivo de este trabajo es medir la prevalencia de caries, enfermedad periodontal y maloclusión en la población escolar de Ceuta, y su distribución según edad, género, etnia y nivel socioeconómico (NSE) - medido a través de la ocupación paterna-

Métodos: Se realizó una exploración física de la cavidad bucal a una muestra de escolares de 7,12 y 14 años $(n=347)$ del distrito sanitario de Ceuta con el fin de calcular los índices cao —dentición temporal-, $C A O$ —dentición definitiva-, CPITN y de maloclusión. La selección de la muestra se realizó de forma aleatoria, estratificada polietápica. Se valoró la significación estadística de las diferencias encontradas aplicando las pruebas de Chi-cuadrado, T de Student. y F de Snedecor. Se calcularon las razones de ventaja (Odds Ratio), según sexo, etnia y NSE, de un índice $C A O$ superior a la mediana de la distribución en escolares de 12 y 14 años.

Resultados: El índice cao (piezas cariadas, ausentes y obturadas en dentición temporal) es 3,02 a los 7 años y el índice $C A O$ (piezas cariadas, ausentes y obturadas en dentición definitiva) es 3,91 a los 12 años y 4,46 a los 14 años. Las razones de ventaja (OR) de un índice $C A O>4$ a los 12-14 años y sus intervalos de confianza (IC) son los siguientes: 2,26 según género $\left(I_{95}=1,27-4,05\right), 2,17$ según etnia $\left(I_{95 \%}=1,18-3,99\right)$ y 1,80 según NSE ( $\left.\mathrm{IC}_{95}=0,85-3,81\right)$. En el estrato de bajo NSE la OR por etnia es $1,38\left(\mathrm{IC}_{95}=0,28-7,0\right)$. No se observan diferencias significativas en la distribución de maloclusión ni de enfermedad periodontal —excepto por edad-.

Conclusiones: Los valores del índice $\mathrm{CAO}$ en escolares de Ceuta son más elevados que el promedio nacional y superiores al objetivo marcado por la OMS para el año 2000. El riesgo de índice CAO superior a la mediana es en niñas 2,3 veces mayor que en niños, en musulmanes 2,17 veces mayor que en no musulmanes y en escolares con bajo nivel socioeconómico - padres desempleados- 1,8 veces mayor que en escolares con padres activos. El aumento de riesgo asociado a etnia está influenciado por el nivel socioeconómico.

Palabras clave: Adolescencia. Salud bucodental. Índice cao. Índice CAO. Desigualdades en salud.

\section{Correspondencia}

Adoración Nieto

Facultad de Medicina. Departamento de Ciencias Socio-sanitarias

Avda Sánchez Pizjuán s/n

41009 Sevilla, Spain

Correo electrónico: adora@cica.es

\section{ABSTRACT}

\section{Oral Health of Schoolchildren in Ceuta.} Influences of Age, Sex, Ethnic

\section{Background and Socioeconomic Level}

Background: The objective of this study is that of gauging the prevalence of dental caries, periodontal disease and malocclusion among the school-age population of Ceuta and the spread thereof by age, sex, ethnic background and father's occupation.

Methods: Prevalence survey of a multistage, stratified, random sample of schoolchildren 7,12 and 14 years of age $(n=347)$ in order to calculate DMFT and dmft indexes - permanent and deciduous dentition-, CPITN and malocclusion. Assessment of the statistical significance of the differences encountered according to age, sex, ethnic background and socioeconomic status using the Chi-Square, Student T and Snedecor F tests. To calculate, among schoolchildren ages 12 and 14 , the odds ratios of showing a DMFT index over 4 and their $95 \%$ confidence intervals by gender, ethnic background and the socioeconomic status.

Results: Dmft (decayed, filled and missing teeth) was 3.02 at 7 and DMFT was 3.91 at 12 and 4.46 at 14 . Odds ratios of DMFT $>4$ were 2.26 by gender $(95 \% \mathrm{CI}=1,27-4,05), 2.17$ by ethnic group $(95 \% \mathrm{CI}=1,18-3,99)$ and 1.8 by SES $(95 \%$ $\mathrm{CI}=0,85-3,81)$. In schoolchildren of low SES - unemployed father's- OR by ethnic group was $1,37(95 \% \mathrm{CI}=0,28-7,0)$. No statistically significant differences were found in the distribution of malocclusion and periodontal disease - except by age.

Conclusions: Dmft and DMFT values are in Ceuta higher than the mean national values and the proposed value of the WHO for the year 2000. Risk of DMFT higher than the median is 2.3 in girls compared with boys, 2.17 in Moslem compared with non Moslem and 1.8 in low SES schoolchildren - unemployed father's - compared to other SES schoolchildren. The increased risk related to ethnic background is influenced by SES.

Keywords: Oral health. DMFT index. dmft index. Health-related inequalities. Early childhood. 


\section{INTRODUCCIÓN}

La caries dental tiene una etiología multifactorial en la que intervienen características del huésped (saliva y esmalte dental), de la flora bucal (placa bacteriana) y del substrato sobre el que ésta se desarrolla (higiene oral y dieta $)^{1}$.

A la importancia de mantener cifras bajas de prevalencia de caries dental se ha sumado recientemente en los países desarrollados la preocupación por el control de las enfermedades periodontales en adultos y ancianos, así como la corrección de la maloclusión en todas las edades.

En España se han realizado cuatro encuestas nacionales de salud oral, la primera en 1968-1969 y la última en 1994, en la que se incluyó un grupo de adultos y otro de ancianos además de las muestras de población escolar $^{2-5}$. Los resultados de estos cuatro estudios en escolares muestran la evolución de los índices de salud oral en nuestro país desde niveles bajos de caries, escaso consumo de azúcar refinado y abundante de legumbres, verduras y frutas frescas (1968-69), evolucionando a cifras de caries más elevadas aunque menores que la de otros países de nuestro entorno, pero con bajos índices de restauración (1983) y más tarde a cifras moderadas de caries y riesgo moderado a alto de enfermedad periodontal, así como un incremento de los índices de restauración, aunque el $40 \%$ de los escolares seguían sin acudir nunca al estomatólogo (1987) y, por último, la confirmación del descenso de la caries dental en nuestro país (1994).

A estos estudios nacionales se suman encuestas de salud oral en comunidades autónomas e incluso en demarcaciones municipales, realizadas como fase previa al establecimiento de medidas preventivas comunitarias y al seguimiento de sus influencias sobre la salud oral ${ }^{6}$.

Este estudio persigue precisamente ese objetivo de medición de la salud oral en los escolares del distrito sanitario de atención primaria de Ceuta.

\section{MATERIAL Y MÉTODOS}

El distrito sanitario de atención primaria de Ceuta atiende a unos 75.000 habitantes distribuidos en tres zonas básicas de salud. Se procedió a un muestreo estratificado polietápico, siendo las unidades de primera etapa (UPE) los centros educativos (CE) del distrito, agrupados según indicadores socioeconómicos y distribuyendo la población proporcionalmente según género y etnia, en base a las estadísticas oficiales del curso $1995-96^{7}$ para asegurar que la media muestral fuera un estimador no sesgado de la media poblacional ${ }^{8}$.

Dentro de cada colegio se seleccionó a los escolares a partir de los listados oficiales de alumnos. La exploración física de la cavidad bucal se realizó a la totalidad de los escolares de las aulas que incluían alumnos de 7, 12 y 14 años, pero sólo se introdujo en la base de datos y se utilizó en el análisis posterior la información procedente de los alumnos seleccionados para la muestra. Cuando alguno de los alumnos de la muestra no había acudido al colegio el día de la exploración era substituido por el siguiente alumno de la lista de clase con igual edad, género y etnia.

La variación esperada sobre la media del índice CAOD se estimó a partir de un estudio piloto previo ${ }^{9}$, admitiéndose un error absoluto máximo de $+-0,2$ a los 7 años, $+-0,5$ a los 12 años y +-0,7 a los 14 años y fijándose el nivel de confianza en el 95\%.

El trabajo de campo se realizó del 15 de mayo al 5 de junio de 1996. Un único examinador, estomatólogo de la consulta de odontología de atención primaria, realizó todas las exploraciones, tras entrenamiento previo con los pacientes menores de 15 años que acudían a dicha consulta. El tiempo medio de exploración de cada escolar estuvo en torno a los cuatro minutos, utilizándose 
guantes de un solo uso y material esterilizado individual —espejos planos del número 5 , sondas de caries afiladas curvas y sondas periodontales - así como gasas de algodón estéril para limpiar y secar los dientes. Una única enfermera de la consulta de odontología de atención primaria anotó los resultados de las exploraciones en las fichas abreviadas de la OMS para encuestas de salud orall $^{10}$, en las que además se recogió la edad, sexo, grupo étnico — según los apellidos y en casos dudosos, como por ejemplo padre y madre con diferente origen, por elección propia del niño- y ocupación paterna, distinguiendo 7 grupos de actividad económica que más tarde se agruparon en 3 estratos: no manual, manual y no activo.

La presencia de caries se midió por pieza dental, elaborándose los índices cao: dientes cariados, ausentes y obturados temporales; CAO: dientes cariados, ausentes y obturados definitivos; y CAOM6: primeros molares cariados, ausentes y obturados.

Para la evaluación de la salud periodontal se utilizó el CPITN ${ }^{11}$, codificándose cada sextante: 0 sano, 1 sangrado al sondaje, 2 cálculo y x excluido; y asignando a cada participante como CPITN global la mayor puntuación obtenida en sus sextantes.

El estado oclusivo se clasificó siguiendo las categorías propuestas por la OMS: 0 «buena oclusión»; 1 «ligera maloclusión» por uno o más dientes rotados o inclinados; leve apiñamiento o espaciamiento que impide la normal alineación; y 2 «moderada o severa maloclusión» por apiñamiento o espaciamiento mayor a $4 \mathrm{~mm}$, resalte en incisivos igual o mayor a $9 \mathrm{~mm}$, mordida cruzada anterior igual o mayor al diámetro anteroposterior de un diente, mordida abierta o desviación de la línea media mayor a 4 $\mathrm{mm}^{10}$.

La significación estadística de las diferencias encontradas por edad, sexo, etnia y ocupación paterna se han calculado para un nivel de confianza del 95\%, usando las prue- bas de Chi-cuadrado para variables cualitativas, la t de Student para la diferencia de medias en variables cuantitativas con dos categorías y la prueba F de Snedecor para variables cuantitativas de 3 categorías ${ }^{12}$, usando el programa SPSS (versión 10.0).

Se han calculado las razones de ventaja (OR) que estiman el riesgo de presentar un índice CAO superior a 4 (que es el valor mediano de la distribución) en escolares de 12 y 14 años, en los diferentes estratos de sexo, grupo étnico y ocupación paterna, así como grupo étnico en dos estratos socioeconómicos diferentes: padres no activos y padres activos (con ocupaciones manuales y no manuales) usando el programa Statcalc incluido en Epi Info ${ }^{13}$.

\section{RESULTADOS}

La tabla 1 muestra la descripción de las características de los escolares de la muestra según edad, sexo, etnia y ocupación de los padres. Los estratos de edad no difieren significativamente en su distribución por sexo - aunque existe un ligero predominio de varones - pero sí en su distribución por etnia y ocupación paterna, ya que los porcentajes de escolares de origen musulmán y padres desempleados disminuyen con la edad.

La tabla 2 muestra la presencia y ausencia de dientes cariados, ausentes y obturados tanto en dentición temporal (índice cao) como permanente (índice CAO y CAOM6). Se observan diferencias estadísticamente significativas por grupo étnico en los tres índices comparados, y por ocupación paterna tan sólo en dentición temporal a los 7 años de edad.

La tabla 3 muestra el promedio de dientes cariados, ausentes y obturados en dentición temporal (índice cao) y definitiva (indices CAO y CAOM6) y su desviación estándar. Se han excluido de esta tabla los valores cao a los 12 y 14 años, así como los índices CAO y CAOM6 a los 7 años por motivos obvios de cronología de recambio dentario, 
Tabla 1

Distribución de los escolares según edad, género, etnia y ocupación paterna. Ceuta, 1996

\begin{tabular}{|lcccccccc|}
\hline Edad & \multicolumn{2}{c}{7 años } & \multicolumn{2}{c}{12 años } & \multicolumn{2}{c}{14 años } & \multicolumn{2}{c|}{ Total } \\
\hline Género & $n$ & $\%$ & $n$ & $\%$ & $n$ & $\%$ & $n$ & $\%$ \\
\hline Niños & 63 & 52,1 & 76 & 53,5 & 43 & 51,2 & 182 & 52,4 \\
Niñas & 58 & 47,9 & 66 & 46,5 & 41 & 48,8 & 165 & 47,6 \\
Grupo étnico & & & & & & & & \\
Musulmán & 50 & 41,3 & 53 & 37,3 & 20 & 23,8 & 123 & 35,5 \\
No musulmán & 71 & 58,7 & 89 & 62,7 & 64 & 76,2 & 224 & 64,5 \\
Ocupación & & & & & & & & 72 \\
No activos & 33 & 27,3 & 27 & 19,0 & 12 & 14,3 & 20,8 \\
Manual & 56 & 46,3 & 59 & 41,6 & 35 & 41,7 & 150 & 43,2 \\
No manual & 32 & 26,4 & 56 & 39,4 & 37 & 44,0 & 125 & 36,0 \\
\hline Totales & 121 & 34,9 & 142 & 40,9 & 84 & 24,2 & 347 & 100 \\
\hline
\end{tabular}

Tabla 2

Ausencia y presencia de dientes cariados, ausentes y obturados temporales (cao), definitivos (CAO) y primeros molares (CÄOM6). Ceuta, 1996

\begin{tabular}{|c|c|c|c|c|c|c|}
\hline & \multirow{2}{*}{\multicolumn{2}{|c|}{$\frac{\text { Escolares de } 7 \text { años }}{\text { Índice cao }}$}} & \multicolumn{4}{|c|}{ Escolares de 12 y 14 años } \\
\hline & & & \multicolumn{2}{|c|}{ Índice $C A O$} & \multicolumn{2}{|c|}{ Índice CAOM6 } \\
\hline & $\begin{array}{c}c a o=0 \\
n(\%)\end{array}$ & $\begin{array}{c}c a o>0 \\
n(\%)\end{array}$ & $\begin{array}{c}C A O=0 \\
n(\%)\end{array}$ & $\begin{array}{c}C A O>0 \\
n(\%)\end{array}$ & $\begin{array}{c}C A O M 6=0 \\
n(\%)\end{array}$ & $\begin{array}{c}\text { CAOM6>0 } \\
n(\%)\end{array}$ \\
\hline \multicolumn{7}{|l|}{ Género } \\
\hline Niños & $24(38,1)$ & $39(61,9)$ & $21(17,6)$ & $98(82,4)$ & $29(24,4)$ & $90(75,6)$ \\
\hline Niñas & $19(32,8)$ & $39(67,2)$ & $15(14,0)$ & $92(86,0)$ & $23(21,5)$ & $84(78,5)$ \\
\hline Valor de $p$ & & 0,540 & & 0,457 & & 0,608 \\
\hline \multicolumn{7}{|l|}{ Etnia } \\
\hline Musulmán & $8(16,0)$ & $42(84,0)$ & $5 \quad(6,8)$ & $68(93,2)$ & $10(13,7)$ & $63(86,3)$ \\
\hline No musulmán & $35(49,3)$ & $36(50,7)$ & $31(20,3)$ & $122(79,7)$ & $42(27,5)$ & $111(72,5)$ \\
\hline Valor de $p$ & & 0,000 & & 0,010 & & 0,022 \\
\hline \multicolumn{7}{|c|}{ Ocupación paterna } \\
\hline No activos & $8(24,2)$ & $25(75,8)$ & $3(7,7)$ & $36(92,3)$ & $7(17,9)$ & $32(82,1)$ \\
\hline Manual & $16(28,6)$ & $40(71,4)$ & $17(18,1)$ & $77(81,9)$ & $23(24,5)$ & $71(75,5)$ \\
\hline No manual & $19(59,4)$ & $13(40,6)$ & $16(17,2)$ & $77(82,8)$ & $22(23,7)$ & $71(76,3)$ \\
\hline Valor de $p$ & & 0,004 & & 0,299 & & 0,705 \\
\hline
\end{tabular}

que reduce las posibilidades de interpretación de dichos índices a esas edades. Las diferencias son estadísticamente significativas por etnia excepto en el grupo de 14 años y por ocupación paterna sólo en dentición temporal.

La tabla 4 muestra los riesgos de presentar un índice CAO superior a 4 asociado al sexo femenino, la etnia musulmana y la inactividad laboral en los padres, así como la etnia musulmana dentro de cada categoría - activo y no activo - de ocupación paterna. Las razones de ventaja (OR) superan el valor de nulidad y son estadísticamente significativas en niñas y escolares musulmanes. Las diferencias de riesgo según la etnia se mantienen en el estrato de escolares con 
Tabla 3

Promedio de dientes cariados, ausentes y obturados temporales y permanentes según edad, género, grupo étnico y ocupación paterna. Ceuta, 1996

\begin{tabular}{|c|c|c|c|c|c|}
\hline \multirow{2}{*}{ Edad } & \multirow{2}{*}{$\begin{array}{c}7 \text { años } \\
\text { cao }(d s)\end{array}$} & \multicolumn{2}{|c|}{12 años } & \multicolumn{2}{|c|}{14 años } \\
\hline & & $C A O(d s)$ & CAOM6 (ds) & $C A O(d s)$ & CAOM6 (ds) \\
\hline \multicolumn{6}{|l|}{ Género } \\
\hline Niños & $2,98(3,42)$ & $3,49(2,67)$ & $2,38(1,58)$ & $3,95(3,36)$ & $2,09(1,64)$ \\
\hline Niñas & $3,09(3,12)$ & $4,39(3,78)$ & $2,36(1,56)$ & $5,00(3,55)$ & $2,54(1,58)$ \\
\hline Valor de $\mathrm{p}$ & 0,865 & 0,098 & 0,946 & 0,169 & 0,212 \\
\hline \multicolumn{6}{|l|}{ Etnia } \\
\hline Musulmán & $4,40(3,46)$ & $5,00(3,26)$ & $2,89(1,33)$ & $5,30(3,44)$ & $2,60(1,60)$ \\
\hline No musulmán & $2,07(2,77)$ & $3,26(3,08)$ & $2,07(1,62)$ & $4,20(3,47)$ & $2,22(1,63)$ \\
\hline Valor de $\mathrm{p}$ & 0,000 & 0,002 & 0,002 & 0,220 & 0,362 \\
\hline \multicolumn{6}{|l|}{ Ocupación } \\
\hline No activo & $3,97(3,46)$ & $4,48(3,96)$ & $2,41(1,51)$ & $6,00(3,54)$ & $3,00(1,48)$ \\
\hline Manual & $3,41(3,38)$ & $4,03(3,30)$ & $2,42(1,56)$ & $4,51(3,84)$ & $2,23(1,65)$ \\
\hline No manual & $1,41(2,20)$ & $3,50(2,82)$ & $2,30(1,61)$ & $3,92(2,99)$ & $2,16(1,62)$ \\
\hline Valor de $\mathrm{p}$ & 0,003 & 0,408 & 0,913 & 0,197 & 0,279 \\
\hline Total & $3,02(3,27)$ & $3,91(3,25)$ & $2,37(1,56)$ & $4,46(3,47)$ & $2,31(1,62)$ \\
\hline
\end{tabular}

Tabla 4

Riesgo de índice CAO superior a la mediana en escolares de 12 y 14 años según género, grupo étnico y ocupación paterna. Ceuta, 1996

\begin{tabular}{|c|c|c|c|c|c|}
\hline & $C A O>4$ & $C A O<=4$ & $O R$ & IC $95 \%$ & Valor de $p$ \\
\hline \multicolumn{6}{|l|}{ Género } \\
\hline Niñas & 53 & 54 & 2,26 & $1,27-4,05$ & 0,003 \\
\hline Niños & 36 & 83 & $1,0^{1}$ & & \\
\hline \multicolumn{6}{|l|}{ Etnia } \\
\hline Musulmana & 38 & 35 & 2,17 & $1,18-3,99$ & 0,007 \\
\hline No musulmana & 51 & 102 & $1,0^{1}$ & & \\
\hline \multicolumn{6}{|l|}{ Ocupación paterna } \\
\hline Padre no activo & 20 & 19 & 1,80 & $0,85-3,81$ & 0,095 \\
\hline Padre activo & 69 & 118 & $1,0^{1}$ & & \\
\hline \multicolumn{6}{|l|}{ Padres no activos } \\
\hline Musulmanes & 15 & 13 & 1,38 & $0,28-7,00$ & 0,652 \\
\hline No musulmanes & 5 & 6 & $1,0^{1}$ & & \\
\hline \multicolumn{6}{|l|}{ Padres activos } \\
\hline Musulmanes & 23 & 22 & 2,18 & $1,05-4,56$ & 0,024 \\
\hline No musulmanes & 46 & 96 & $1,0^{1}$ & & \\
\hline
\end{tabular}

${ }^{1}$ Categoría de referencia 
padres activos y desaparecen en el estrato de escolares con padres desempleados.

La tabla 5 muestra la distribución del índice CPITN, que cuantifica la presencia o ausencia de enfermedad periodontal, según edad, sexo, grupo étnico y ocupación paterna. Se observan diferencias estadísticamente significativas por edad (a mayor edad menor proporción de escolares con CPITN 0, que significa ausencia de enfermedad periodontal), pero no por el resto de las variables.

Tabla 5

Enfermedad periodontal según edad, género, etnia y profesión paterna en escolares de Ceuta, 1996

\begin{tabular}{|c|c|c|c|c|c|c|}
\hline & \multicolumn{2}{|c|}{ CPITN 0} & \multicolumn{2}{|c|}{ CPITN 1} & \multicolumn{2}{|c|}{ CPITN 2} \\
\hline & $n$ & $\%$ & $n$ & $\%$ & $n$ & $\%$ \\
\hline \multicolumn{7}{|l|}{ Edad } \\
\hline 7 años & 87 & 71,9 & 24 & 19,8 & 10 & 8,3 \\
\hline 12 años & 76 & 53,5 & 26 & 18,3 & 40 & 18,2 \\
\hline 14 años & 41 & 48,8 & 12 & 14,3 & 31 & 36,9 \\
\hline Valor de $\mathrm{p}$ & & & & & & 0,000 \\
\hline \multicolumn{7}{|l|}{ Género } \\
\hline Niños & 99 & 54,4 & 32 & 17,6 & 51 & 28,0 \\
\hline Niñas & 105 & 63,6 & 30 & 18,2 & 30 & 18,2 \\
\hline Valor de $\mathrm{p}$ & & & & & & 0,088 \\
\hline \multicolumn{7}{|l|}{ Grupo étnico } \\
\hline Musulmán & 64 & 52,0 & 27 & 22,0 & 32 & 26,0 \\
\hline No musulmán & 140 & 62,5 & 35 & 15,6 & 49 & 21,9 \\
\hline Valor de $\mathrm{p}$ & & & & & & 0,146 \\
\hline \multicolumn{7}{|l|}{ Ocupación paterna } \\
\hline Padres no activos & 41 & 56,9 & 17 & 23,6 & 14 & 19,4 \\
\hline Manual & 86 & 57,3 & 24 & 16,0 & 40 & 26,7 \\
\hline No manual & 77 & 61,6 & 21 & 16,8 & 27 & 21,6 \\
\hline Valor de $\mathrm{p}$ & & & & & & 0,508 \\
\hline
\end{tabular}

La tabla 6 muestra la distribución de maloclusión según edad, género, grupo étnico y ocupación paterna, sin que observemos diferencias estadísticamente significativas en ninguna de estas variables.

\section{DISCUSIÓN}

Al observar las características de la muestra de escolares según edad, sexo, etnia y ocupación paterna, llama la atención el mayor porcentaje de escolares de etnia musulmana en los estratos de edad más jóvenes. Este hecho podría deberse a la nacionalización en 1986 de un colectivo importante de ciudadanos de origen marroquí y a la mayor natalidad de este grupo étnico ${ }^{14}$ que podría haber conducido a un progresivo incremento de su peso relativo en sucesivas cohortes de nacimiento.

También podría indicar que al aumentar la edad disminuye el cumplimiento de la escolarización obligatoria en el colectivo musulmán, lo cual sólo podría comprobarse mediante el cruce de los datos de padrón municipal y de la delegación de Educación.

La desigual distribución según ocupación paterna en los diferentes estratos de edad, especialmente al comparar los escolares de 
Tabla 6

Maloclusión en escolares según edad, género, grupo étnico y ocupación paterna. Ceuta 1996

\begin{tabular}{|c|c|c|c|c|c|c|}
\hline & \multicolumn{2}{|c|}{ Buena oclusión } & \multicolumn{2}{|c|}{ Maloclusión ligera } & \multicolumn{2}{|c|}{$\begin{array}{l}\text { Maloclusión moderada o } \\
\text { grave }\end{array}$} \\
\hline & $n$ & $\%$ & $n$ & $\%$ & $n$ & $\%$ \\
\hline \multicolumn{7}{|l|}{ Edad } \\
\hline 7 años & 15 & 12,4 & 78 & 64,5 & 28 & 23,1 \\
\hline 12 años & 22 & 15,5 & 85 & 59,9 & 35 & 24,6 \\
\hline 14 años & 8 & 9,5 & 60 & 71,4 & 16 & 19,0 \\
\hline Valor de $\mathrm{p}$ & & & & & & 0,503 \\
\hline \multicolumn{7}{|l|}{ Género } \\
\hline Niños & 18 & 9,9 & 127 & 69,8 & 37 & 20,3 \\
\hline Niñas & 27 & 16,4 & 96 & 58,2 & 42 & 25,5 \\
\hline Valor de $\mathrm{p}$ & & & & & & 0,061 \\
\hline \multicolumn{7}{|l|}{ Grupo étnico } \\
\hline Musulmán & 15 & 12,2 & 73 & 59,3 & 35 & 28,5 \\
\hline No musulmán & 30 & 13,4 & 150 & 67,0 & 44 & 19,6 \\
\hline Valor de $\mathrm{p}$ & & & & & & 0,173 \\
\hline \multicolumn{7}{|l|}{ Ocupación paterna } \\
\hline Padres no activos & 11 & 15,3 & 43 & 59,7 & 18 & 25,0 \\
\hline Manual & 22 & 14,7 & 94 & 62,7 & 34 & 22,7 \\
\hline No manual & 12 & 9,6 & 86 & 68,8 & 27 & 21,6 \\
\hline Valor de $\mathrm{p}$ & & & & & & 0,618 \\
\hline
\end{tabular}

7 y 14 años, posiblemente esté a su vez influenciada por esta diferente distribución por grupo étnico.

En cualquier caso, la distribución de la muestra, en función del método de muestreo elegido, es un reflejo de las características sociodemográficas de la población escolarizada en el distrito de salud de Ceuta.

Los índices encontrados de caries dental, comparados con los índices nacionales ${ }^{2-5}$, son claramente desfavorables. El único valor nacional de índice $\mathrm{CAO}$ a los 12 años superior al de los escolares de Ceuta es el de la encuesta de $1983^{3}$ ), mientras que nuestro índice es casi un $60 \%$ superior al más reciente, obtenido en $1994^{5}$ lo que, de acuerdo con la escala propuesta por la OMS, sitúa los resultados nacionales en un estrato de baja prevalencia de caries y los de Ceuta en la banda alta del estrato de prevalencia moderada ${ }^{15}$.

Cuando comparamos con los valores promedios de comunidades vecinas, como los de Andalucía en 1995, el índice CAO en Ceuta es un $45 \%$ más alto y, además, la proporción de escolares de 12 años con índice CAO mayor o igual a 10 es $6,3 \%$ mientras que en Andalucía era sólo del 1\% ${ }^{16}$.

Nos encontramos por tanto con un grave problema de patología dental escolar, de mayor magnitud en la población de procedencia musulmana y en escolares con padres no activos independientemente de su grupo étnico. Estos estratos poblacionales no son minoritarios, de hecho los escolares musulmanes suponen más de un tercio de la muestra y los hijos de padres desempleados son una quinta parte de la misma, aunque estas dos variables no son independientes, ya que el $77,8 \%$ de los escolares de padres no activos son de origen musulmán, siendo estadísticamente significativas las diferencias en la distribución simultanea de la muestra según ocupación paterna y grupo étnico.

Los resultados parecen describir una afectación por caries con una distribución de- 
pendiente del nivel socioeconómico familiar, puesto que cuando éste es bajo - padres desempleados - no existen diferencias étnicas. Algunas publicaciones recientes ya han enfatizado la importancia del nivel socioeconómico (medido a través de la ocupación, el área geográfica de residencia, el salario familiar, etc.) sobre la aparición y el mantenimiento de caries dental ${ }^{17,18}$. De hecho el primer informe estadounidense sobre salud oral incide en la desigual distribución del descenso de la caries dental y encuentra diferencias significativas en la prevalencia de caries en dientes temporales y en los índices de restauración tanto de dentición temporal —ir- como permanente $-I R-{ }^{19}$. Estos resultados son equivalentes a los encontrados en Ceuta ya que la distribución de dientes obturados respecto a los cariados, ausentes y obturados (índices $i r$, IR) que no se presenta en tablas, es marcadamente diferente según ocupación paterna; el índice ir a los 7 años es 2,71 pero en escolares de padres no activos es 0,00 y el índice $I R$ a los 12 y 14 años es 9,97 y 19,06 pero en escolares de padres no activos es 0,00 y 5,50 .

Las posibilidades de prevención deberían, por tanto, tener un componente comunitario que neutralizara parcialmente las influencias del nivel socioeconómico y cultural ${ }^{20}$. En estas circunstancias probablemente la primera actuación comunitaria debería ser la fluoración de las aguas de bebida, medida que a su probada eficacia une la ventaja de no requerir participación individualizada alguna $^{21-24}$ y que actualmente no se realiza más que en un sector reducido de la población total abastecida.

Además sería muy interesante poder disponer de un programa de aplicación de colutorios fluorados a escolares, como los que han ayudado a reducir la prevalencia de caries en países de nuestro entorno ${ }^{25}$, así como insistir en lo básico dentro de los contenidos curriculares de educación para la salud, es decir, proporcionar información pero también adiestramiento en las técnicas de higiene oral así como cepillos y dentífricos fluo- rados a los escolares, con la participación de sus profesores.

Podría ser efectivo ampliar más tarde estas actividades a madres de niños en edad escolar y a mujeres embarazadas, contando con la participación de pediatras y diplomados en enfermería, programas preventivos sobre los que ya existen precedentes ${ }^{26}$.

Respecto a las actividades propiamente curativas y reparadoras, se debería potenciar la aplicación de selladores en las consultas de odontología de atención primaria, así como ampliar la asistencia actualmente proporcionada (únicamente exodóntica) a los tratamientos de obturación, comenzando, en función de los resultados presentados en las tablas, por los primeros molares permanentes.

\section{BIBLIOGRAFÍA}

1. Reich E, Lussi A, Newbrun E. Caries-risk assessment. Int Dent J 1999; 49: 15-26.

2. Gimeno de Sande A, Sánchez B, Viñes JJ, Gómez F, Mariño F. Estudio epidemiológico de la caries dental y patología bucal en España. Rev Sanid Hig Pública1971; 45:361-433.

3. Cuenca E. La encuesta de la OMS sobre la salud buco-dental en España. Una aproximación personal. Arch Odonto Estomatol 1986; 2:15-22.

4. Sicilia A, Cobo J, Noguerol B et al. Prevalencia de caries en los niños y jóvenes escolares españoles de 7, 12 y 15-19 años. Av Odontoestomatol 1990; 6: 323-330.

5. Noguerol B, Llodra JC, Sicilia A, Follana M. La salud bucodental en España. 1994. Antecedentes y perspectivas de futuro. Madrid: Avances; 1995.

6. González Serrano A. Estudios epidemiológicos de caries y razón costo-beneficio después de tres años de fluoración de El Pedroso (Sevilla). [Tesis Doctoral]. Sevilla: Facultad de Medicina. Universidad de Sevilla. Sevilla; 1984.

7. Dirección Provincial del Ministerio de Educación y Ciencia. Datos estadísticos del curso 1995-96. Ceuta: MEC; 1996.

8. Silva LC. Muestreo para la investigación en ciencias de la salud. Madrid: Díaz de Santos; 1993. 
9. Nieto MM, Nieto V. Diagnóstico de salud oral y educación para la salud bucodental en un grupo de escolares ceutíes. Transfetana (Inst Estudios Ceutíes) 1997; 3 (Sanidad pública en Ceuta): 83-92.

10. WHO. Oral Health Surveys: Basic Methods. $3^{\text {rd }}$ ed. Geneva: WHO; 1984.

11. Cutress TW, Ainamo J, Sardo-Infirri J. The community periodontal index of treatment needs (CPITN) procedure for population groups and individuals. Int Dent J 1987; 37:222-3.

12. Armitage P, Berry G. Estadística para la investigación biomédica. 3. ${ }^{a}$ edición. Barcelona: Harcourt Brace; 1997.

13. Dean JA, Dean AG, Burton A, Dicker R, Brendel KA. Statcalc. Division of surveillance and epidemiologic studies. Epidemiology program office. Atlanta: CDC; 1990.

14. Nieto A. Madres y recién nacidos: análisis epidemiológico de las características sanitarias más relevantes. [Tesina de Diplomado en Sanidad] Madrid: Escuela Nacional de Sanidad. Ceuta; 1991.

15. WHO. Oral health for the 21 st century. Oral Health Unit. Geneva: WHO; 1993.

16. Llodra JC. Estudios epidemiológicos de salud bucodental. Resultados y controversias. Ponencia en las II Jornadas Andaluzas de Salud Oral en Atención Primaria. Sanlúcar de Barrameda; 1997.

17. Faggiano F, Stanislao FD, Lemma P, Renga G. Role of social class in caries occurrence in 12 year olds in Turin, Italy. Eur J Public Health 1999, 9:109-113.

18. Eckersley AJ, Blinkhorn FA. Dental attendance and dental health behaviour in children from deprived and non-deprived areas of Salford, north-west England. Int J Paediatr Dent 2001; 11: 103-109.

19. Ismail AI, Sohn W. The impact of universal access to dental care on disparities in caries experience in children. J AM Dent Assoc 2001; 132: 295-303.

20. Gillcrist JA, Brumley DE, Blackford JU. Community socioeconomic status and children's dental health. J Am Dent Assoc 2001; 132: 216-222.

21. Burt BA. Fifty years of water fluoridation. $\mathrm{Br}$ Dent J 1995; 21:49-50.

22. Análisis coste-beneficio de la fluoración de las aguas de abastecimiento público de la ciudad de Málaga. Gac Sanit 1991; 5:82-86.

23. Salleras L, Bohigas L, Cuenca E, Martínez-Carretero JM, Manau C. Análisis coste-efectividad de tres programas comunitarios alternativos de uso preventivo del flúor para la prevención de la caries dental en Cataluña. Rev Esp Estomatol 1988; 4: 291-302.

24. Marín MD, Pacheco F, Martínez R, Gómez E, Gutierrez C. La fluoración de las aguas en la región de Murcia. Rev Esp Salud Pública 1998; 72: 91-101.

25. Haugejorden O. Using the DMF gender difference to assess the «major» role of fluoride toothpastes in the caries decline in industrialized countries: a meta-analysis. Community Dent Oral Epidemiol 1996; 24: 369-75.

26. Lewis CW, Grossman DC, Domoto PK, Deyo RA. The role of pediatrician in the oral health of children: A national survey. Pediatrics 2000; 106:E84. 